Pacific Journal of Mathematics

ON BANACH SPACE VALUED EXTENSIONS FROM SPLIT 


\section{ON BANACH SPACE VALUED EXTENSIONS FROM SPLIT FACES}

\section{TAge BaI ANDERSEN}

The object of this note is the following theorem: Suppose $a$ is a continuous affine map from a closed split face $F$ of a compact convex set $K$ with values in a Banach space $B$ enjoying the approximation property. Suppose also that $p$ is a strictly positive lower semi-continuous concave function on $K$ such that $\|a(k)\| \leqq p(k)$ for all $k$ in $F$. Then $a$ admits a continuous affine extension $\tilde{a}$ to $K$ into $B$ such that $\|\tilde{a}(k)\| \leqq p(k)$ for all $k$ in $K$.

We shall use the methods of tensor products of compact convex sets as developed by Semadeni [12], Lazar [9], Namioka and Phelps [10] and Behrends and Wittstock [6] to reduce the problem to the case $B=R$, and in this case the result follows from the work of Alfsen and Hirsberg [3] and the present author [4].

We shall be concerned with compact convex sets $K_{1}$ and $K_{2}$ in locally convex spaces $E_{1}$ and $E_{2}$ respectively. By $A\left(K_{i}\right)$ we shall denote the continuous real affine functions on $K_{i}$ for $i=1,2$. We let $B A\left(K_{1} \times K_{2}\right)$ be the Banach space of continuous biaffine functions on $K_{1} \times K_{2}$. We observe that $1 \in B A\left(K_{1} \times K_{2}\right)$ and that $B A\left(K_{1} \times K_{2}\right)$ separates points of $K_{1} \times K_{2}$. As usual we define the projective tensor product of $K_{1}$ and $K_{2}, K_{1} \otimes K_{2}$, to be the state space of $B A\left(K_{1} \times K_{2}\right)$ equipped with the $w^{*}$-topology. Then $K_{1} \otimes K_{2}$ is a compact convex set, and we have a homeomorphic embedding $\omega_{K_{1} \times K_{2}}$ (called $\omega$, when no confusion can arise) from $K_{1} \times K_{2}$ into $K_{1} \otimes K_{2}$ defined by the following rule: For all $a$ in $B A\left(K_{1} \times K_{2}\right)$ and all $\left(x_{1}, x_{2}\right)$ in $K_{1} \times K_{2}$

$$
\omega\left(x_{1}, x_{2}\right)(a)=a\left(x_{1}, x_{2}\right) \text {. }
$$

We notice that $\omega$ is a biaffine map. It was proved in [10; Prop. 1.3, Th. 2.3] and [6; Satz 1.1.3] that $\partial_{e}\left(K_{1} \otimes K_{2}\right)=\omega\left(\partial_{e} K_{1} \times \partial_{e} K_{2}\right)$, where in general we denote the extreme points of a convex set $K$ by $\partial_{e} K$.

For $a$ in $A\left(K_{1}\right)$ and $b$ in $A\left(K_{2}\right)$ we define the continuous biaffine function $a \otimes b$ by

$$
a \otimes b\left(x_{1}, x_{2}\right)=a\left(x_{1}\right) b\left(x_{2}\right), \text { all }\left(x_{1}, x_{2}\right) \in K_{1} \times K_{2} .
$$

We let $A\left(K_{1}\right) \otimes A\left(K_{2}\right)$ be the real vector space

$$
A\left(K_{1}\right) \otimes A\left(K_{2}\right)=\left\{\sum_{i=1}^{n} a_{i} \otimes b_{i} \mid a_{i} \in A\left(K_{1}\right), b_{i} \in A\left(K_{2}\right)\right\}
$$


which is a copy of the algebraic tensor product of $A\left(K_{1}\right)$ and $A\left(K_{2}\right)$. We denote by $A\left(K_{1}\right) \otimes_{\varepsilon} A\left(K_{2}\right)$ the uniform closure of $A\left(K_{1}\right) \otimes A\left(K_{2}\right)$ in $B A\left(K_{1} \times K_{2}\right)$.

We recall that a Banach space $B$ is said to have the approximation property if for each compact convex subset $C$ of $B$ and each $\varepsilon>0$ there is a continuous linear map $T: B \rightarrow B$ such that $T(B)$ is finite dimensional and such that $\|T x-x\|<\varepsilon$ for all $x \in C$. It is proved in [10; Lem. 2.5] that if $A\left(K_{1}\right)$ (or $A\left(K_{2}\right)$ ) has the approximation property then $B A\left(K_{1} \times K_{2}\right)=A\left(K_{1}\right) \otimes_{\varepsilon} A\left(K_{2}\right)$.

Following Lazar [9] we define $T_{1}$ and $T_{2}$ as the natural embeddings of $A\left(K_{1}\right)$ and $A\left(K_{2}\right)$ into $B A\left(K_{1} \times K_{2}\right)$, i.e.

$$
\begin{aligned}
& T_{1} a=a \otimes 1, \text { all } a \in A\left(K_{1}\right) \\
& T_{2} b=1 \otimes b, \text { all } b \in A\left(K_{2}\right) .
\end{aligned}
$$

Let $P_{i}$ be the adjoint map of $T_{i}$ for $i=1,2$. Then $P_{i}$ is an affine and continuous map of $K_{1} \otimes K_{2}$ onto $K_{i}$ (= state space of $A\left(K_{i}\right)$ ), and

$$
P_{i} \omega\left(k_{1}, k_{2}\right)=k_{i}, i=1,2 .
$$

The first part of the following proposition was proved by Lazar in the case where $K_{1}$ and $K_{2}$ are simplexes, but the proof holds in general. The last part was proved by Lazar in the simplex case by means of the Stone-Weierstrass Theorem for simplexes.

Proposition 1. Let $F_{1}$ and $F_{2}$ be closed faces of compact convex sets $K_{1}$ and $K_{2}$ resp. Let $F=P_{1}^{-1}\left(F_{1}\right) \cap P_{2}^{-1}\left(F_{2}\right)$

(i) Then $F$ is a closed face in $K_{1} \otimes K_{2}$ and $F=\overline{\operatorname{co}}\left(\omega\left(F_{1} \times F_{2}\right)\right)$

(ii) If $A\left(F_{1}\right)$ or $A\left(F_{2}\right)$ has the approximation property then $F_{1} \otimes F_{2}$ is affinely homeomorphic to $F$.

Proof. Since $P_{i}$ is continuous and affine it is immediate that $P_{i}^{-1}\left(F_{i}\right)$ is a closed face of $K_{1} \otimes K_{2}$, and hence $F$ is a closed face.

Now let $p=\omega\left(k_{1}, k_{2}\right) \in \omega\left(F_{1} \times F_{2}\right)$. Then $P_{i} p=k_{i} \in F_{i}$, and hence $p \in P_{1}^{-1}\left(F_{1}\right) \cap P_{2}^{-1}\left(F_{2}\right)=F$. By the Krein Milman Theorem: $\overline{c o}\left(\omega\left(F_{1} \times F_{2}\right)\right)$ $\subseteq F$.

Conversely, let $p \in \partial_{e} F$. Since $F$ is a closed face we get

$$
p \in \partial_{e} F=F \cap \partial_{e}\left(K_{1} \otimes K_{2}\right)=F \cap \omega\left(\partial_{e} K_{1} \times \partial_{e} K_{2}\right) .
$$

Hence $p=\omega\left(x_{1}, x_{2}\right), x_{i} \in \partial_{e} K_{i}$. Then $P_{i} p=x_{i}$ belongs to $F_{i}$ by the definition of $F$. Hence $p \in \omega\left(F_{1} \times F_{2}\right)$, and again by the Krein Milman Theorem $F \subseteq \overline{c o}\left(\omega\left(F_{1} \times F_{2}\right)\right)$, and (i) is proved.

Now we shall prove (ii) under the assumption that $A\left(F_{1}\right)$ has the approximation property. We shall define a continuous affine map 
$T: F_{1} \otimes F_{2} \rightarrow K_{1} \otimes K_{2}$ by

$$
(T \varphi)(b)=\varphi\left(\left.b\right|_{F_{1} \times F_{2}}\right), \varphi \in F_{1} \otimes F_{2}, b \in B A\left(K_{1} \times K_{2}\right) .
$$

Then $T\left(F_{1} \otimes F_{2}\right)$ is compact and convex in $K_{1} \otimes K_{2}$. If $\varphi \in \partial_{e}\left(F_{1} \otimes F_{2}\right)$ then $\varphi=\omega_{F_{1} \times F_{2}}\left(x_{1}, x_{2}\right)$, where $x_{i} \in \partial_{e} F_{i}, i=1,2$. But then

$$
(T \varphi)(b)=b\left(x_{1}, x_{2}\right)=\omega_{K_{1} \times K_{2}}\left(x_{1}, x_{2}\right)(b) \text {, all } b \in B A\left(K_{1} \times K_{2}\right) \text {. }
$$

Hence $T \varphi=\omega_{K_{1} \times K_{2}}\left(x_{1}, x_{2}\right) \in \overline{\mathrm{c} 0}\left(\omega_{K_{1} \times K_{2}}\left(F_{1} \times F_{2}\right)\right)=F$. By the Krein Milman Theorem we conclude that $T\left(F_{1} \otimes F_{2}\right) \leqq F$.

Conversely, if $\psi \in \partial_{e} F$ then as $F$ is a closed face, we get by Milman's theorem

$$
\psi \in \omega_{K_{1} \times K_{2}}\left(F_{1} \times F_{2}\right) \cap \omega_{K_{1} \times K_{2}}\left(\partial_{e} K_{1} \times \partial_{e} K_{2}\right)=\omega_{K_{1} \times K_{2}}\left(\partial_{e} F_{1} \times \partial_{e} F_{2}\right) .
$$

If $\psi=\omega_{K_{1} \times K_{2}}\left(x_{1}, x_{2}\right), x_{i} \in \partial_{e} F_{i}$, then $\omega_{F_{1} \times F_{2}}\left(x_{1}, x_{2}\right) \in \partial_{e}\left(F_{1} \otimes F_{2}\right)$, and as above $\psi=T\left(\omega_{F_{1} \times F_{2}}\left(x_{1}, x_{2}\right)\right)$. By the Krein Milman Theorem we get $F \subseteq T\left(F_{1} \otimes F_{2}\right)$, and so $T$ is surjective.

We proceed to show that $T$ is injective. This is the case if $\left.B A\left(K_{1} \times K_{2}\right)\right|_{F_{1} \times F_{2}}$ is dense in $B A\left(F_{1} \times F_{2}\right)$. We show that $\left.A\left(K_{1}\right) \otimes A\left(K_{2}\right)\right|_{F_{1} \times F_{2}}$ is dense in $B A\left(F_{1} \times F_{2}\right)$. Hence let $c \in B A\left(F_{1} \times F_{2}\right)$ and $\varepsilon>0$. Since $A\left(F_{1}\right)$ has the approximation property, we have that $A\left(F_{1}\right) \otimes_{8} A\left(F_{2}\right)=$ $B A\left(F_{1} \otimes F_{2}\right)$, so there exist $a_{1}, \cdots, a_{n} \in A\left(F_{1}\right), b_{1}, \cdots, b_{n} \in A\left(F_{2}\right)$ such that

$$
\left\|c-\sum_{i=1}^{n} a_{i} \otimes b_{i}\right\|_{F_{1} \times F_{2}}<\frac{\varepsilon}{2} .
$$

Now $\left.A\left(K_{i}\right)\right|_{F_{i}}$ is dense in $A\left(F_{i}\right)$, so we can choose $a_{1}^{\prime} \in A\left(K_{1}\right), b_{i}^{\prime} \in A\left(K_{2}\right)$, $i=1, \cdots n$, such that

$$
\left\|\sum_{i=1}^{n} a_{i} \otimes b_{i}-\sum_{i=1}^{n} a_{i}^{\prime} \otimes b_{1}^{\prime}\right\|_{F_{1} \times F_{2}}<\frac{\varepsilon}{2} .
$$

Then $\left\|c-\sum_{i=1}^{n} a_{i}^{\prime} \otimes b_{i}^{\prime}\right\|_{F_{1} \times F_{2}}<\varepsilon$, and the claim follows.

The next step is to prove that $\overline{\mathrm{co}}\left(\omega\left(F_{1} \times F_{2}\right)\right)$ is a closed split face of $K_{1} \otimes K_{2}$ provided $F_{i}$ is a closed split face of $K_{i}$ for $i=1,2$, and f.ex. $A\left(F_{1}\right)$ has the approximation property.

We shall remind the reader of the following definitions and facts: If $F$ is a closed face of a compact convex $K$, then the complementary $\sigma$-face $F^{\prime}$ is the union of all faces disjoint from $F$. It is always true that $K=\operatorname{co}\left(F \cup F^{\prime}\right) . \quad F$ is called a split face if $F^{\prime}$ is a face and each point in $K \backslash\left(F \cup F^{\prime}\right)$ can be decomposed uniquely as convex combination of a point in $F$ and a point in $F^{\prime}$. It follows from a slight modification of the proof of [2; Th. 3.5] that a closed face is a split face if and only if each nonnegative u.s.c. affine function of $F$ admits an u.s.c. 
affine extension to $K$, which is equal to 0 on $F^{\prime}$. This characterization is sometimes inconvenient because of the "nonsymmetric" properties of the affine functions involved. Using the above characterization we shall give a new one involving the space $A_{s}(K)$ which is the smallest uniformly closed subspace of the bounded functions on $K$ containing the bounded u.s.c. affine functions. This space has been used f.ex. by Krause [8] and Behrends and Wittstock [6] in simplex theory and by Combes [7] in $C^{*}$-algebra theory. We shall state some of the known properties of $A_{s}(K)$.

LEMMA 2.

(i) If $a \in A_{s}(K)$ and $a \geqq 0$ on $\partial_{e} K$ then $a \geqq 0$ on $K$.

(ii) If $a \in A_{s}(K)$ then $\|a\|_{K}=\|a\|_{\delta_{e} K}$.

(iii) If $a \in A_{s}(K)$ then a satisfies the barycentric calculus.

Sketch of proof. If $s$ and $t$ are u.s.c. affine functions on $K$ and $s \leqq t$ on $\partial_{e} K$ it follows by [5; Lem. 1] that $s \leqq t$ on $K$. Hence (i) follows by a limit argument. Now (ii) follows by (i), since on $\partial_{e} K:-\|a\|_{\partial_{e} K} \leqq a \leqq\|a\|_{\partial_{e} K}$. Hence the same inequality holds on $K$, and so $\|a\|_{K} \leqq\|a\|_{\partial_{e} K}$. The converse inequality is trivial. Finally (iii) follows from Lebesgue's theorem on dominated convergence, since the barycentric calculus holds for (differences of) u.s.c bounded affine functions, cf. [1; Cor. I. 1.4].

Proposition 3. Let $F$ be a closed face of a compact convex set $K$. Then $F$ is a split face if and only if each $a \in A_{s}(F)\left(\right.$ or $A_{s}(F)^{+}, A(F)$, $\left.A(F)^{+}, A(F ; K), A(F ; K)^{+}\right)$has an extension $\widetilde{a} \in A_{s}(K)$ such that $\widetilde{a}=0$ on $F^{\prime}$. If such an extension exists then it is unique.

Proof. The uniqueness statement follows from Lemma 2 (ii), since $\partial_{e} K \subseteq F \cup F^{\prime}$.

Assume $F$ is a split face and let $a \in A_{s}(F)$. If $a$ is u.s.c. affine and nonnegative $\alpha$ has as noted above an u.s.c. affine extension $\widetilde{a}$ with $\widetilde{a}=0$ on $F^{\prime}$. Hence the result follows if $a$ is the difference of two nonnegative u.s.c. affine functions on $K$. In general there are $b_{n}, c_{n}$ u.s.c. affine and nonnegative, $a_{n}=b_{n}-c_{n}$, such that $\left\|a_{n}-a\right\|_{F_{n \rightarrow \infty}} 0$. We use Lemma 2 (ii) and the fact that $\partial_{e} K \subseteq F \cup F^{\prime}$ to conclude that

$$
\left\|\tilde{a}_{n}-\tilde{a}_{m}\right\|=\left\|\tilde{a}_{n}-\tilde{a}_{m}\right\|_{\partial_{e} K}=\left\|a_{n}-a_{m}\right\|_{\partial_{e} F}=\left\|a_{n}-a_{m}\right\|_{F} \cdot
$$

Hence $\left\{\widetilde{a}_{n}\right\}_{1}^{\infty}$ is Cauchy in $A_{s}(K)$. Then $\widetilde{a}=\lim \widetilde{a}_{n} \in A_{s}(K)$ will be an extension of $a$ with $\widetilde{a}=0$ on $F^{\prime}$.

Conversely, assume that each $a \in A(F ; K)^{+}$has an extension $\widetilde{a} \epsilon$ $A_{s}(K)$ such that $\widetilde{a}=0$ on $F^{\prime}$. Let $x \in K \backslash\left(F \cup F^{\prime}\right), x=\lambda y+(1-\lambda) z$, 
where $y \in F, z \in F^{\prime}$ and $0<\lambda<1$. Then $\lambda=\tilde{\mathbf{1}}(x)$, and since $\lambda$ is uniquely determined, $\hat{\chi}_{F}$ is affine, and hence $F^{\prime \prime}=\hat{\chi}_{F}^{-1}(0)$ is a face, cf. [2; Prop. 1.1, Cor. 1.2]. Now the uniqueness of $F, F^{\prime}$ components is easy, since $A(F ; K)^{+}$separates points of $F$.

The following lemma can be derived from [6; Formula (1), p. 263, Satz 2.1.3]. For the readers convenience we shall give a proof.

LEMMA 4. Let $K_{1}$ and $K_{2}$ be compact convex sets and $a \in A_{s}\left(K_{1}\right)$, $b \in A_{s}\left(K_{2}\right)$. Then there is a function $c \in A_{s}\left(K_{1} \otimes K_{2}\right)$, denoted by $a \otimes$ $b$, such that

$$
c\left(\omega\left(x_{1}, x_{2}\right)\right)=a\left(x_{1}\right) b\left(x_{2}\right), \text { all }\left(x_{1}, x_{2}\right) \in K_{1} \times K_{2} .
$$

Proof. First we shall consider the case where $a$ and $b$ are nonnegative u.s.c. and affine. Then there exist nets $\left\{a_{\alpha}\right\} \subseteq A\left(K_{1}\right)^{+}$, $\left\{b_{\beta}\right\} \subseteq A\left(K_{2}\right)^{+}$such that $a_{\alpha} \searrow a, b_{\beta} \searrow b$, pointwise. Then $\left\{a_{\alpha} \otimes b_{\beta}\right\}$ is a decreasing net in $B A\left(K_{1} \times K_{2}\right)^{+}$, and therefore there is an u.s.c. affine function $c$ on $K_{1} \otimes K_{2}$ such that

$$
c(\varphi)=\inf _{\alpha, \beta} \varphi\left(a_{\alpha} \otimes b_{\beta}\right), \text { all } \varphi \in K_{1} \otimes K_{2} .
$$

Especially, for all $\left(x_{1}, x_{2}\right) \in K_{1} \times K_{2}$

$$
c\left(\omega\left(x_{1}, x_{2}\right)\right)=\inf a_{\alpha}\left(x_{1}\right) b_{\beta}\left(x_{2}\right)=a\left(x_{1}\right) b\left(x_{2}\right) .
$$

If

$$
a=a_{1}-a_{2}, b=b_{1}-b_{2}
$$

where $a_{i}$ is u.s.c. nonnegative and affine on $K_{1}, b_{i}$ is u.s.c. nonnegative and affine on $K_{2}$, then $\left(x_{1}, x_{2}\right) \rightarrow a\left(x_{1}\right) b\left(x_{2}\right)$ is linear combination of four terms of the kind considered in the first part of the proof, and we can choose $c$ as the corresponding linear combination of elements from $A_{s}\left(K_{1} \otimes K_{2}\right)$.

If $a \in A_{s}\left(K_{1}\right), b \in A_{s}\left(K_{2}\right)$ are arbitrary then we can find $a_{n}^{\prime}, b_{n}^{\prime}$ of the type $\left({ }^{*}\right)$, such that $\left\|b-b_{n}^{\prime}\right\|_{K_{2}}<1 / n,\left\|a-a_{n}^{\prime}\right\|_{K_{1}}<1 / n$ and $c_{n} \in$ $A_{s}\left(K \otimes K_{2}\right)$ such that

$$
c_{n}\left(\omega\left(x_{1}, x_{2}\right)\right)=a_{n}^{\prime}\left(x_{1}\right) b_{n}^{\prime}\left(x_{2}\right) \text {, all }\left(x_{1}, x_{2}\right) \in K_{1} \times K_{2} .
$$

Then for all $\left(x_{1}, x_{2}\right) \in \partial_{e} K_{2}$

$$
\left|a\left(x_{1}\right) b\left(x_{2}\right)-c_{n}\left(\omega\left(x_{1}, x_{2}\right)\right)\right|<\frac{1}{n^{2}}+\frac{1}{n}\left(\|a\|_{K_{1}}+\|b\|_{K_{2}}\right) .
$$

From this it follows that $\left\{\left.c_{n}\right|_{\partial_{e}\left(K_{1} \otimes K_{2}\right)}\right\}$ is Cauchy, and hence $\left\{c_{n}\right\}$ is Cauchy on $K_{1} \otimes K_{2}$ by Lemma 2 (ii). Let $c=\lim c_{n} \in A_{s}\left(K_{1} \otimes K_{2}\right)$. Then it is obvious from $\left({ }^{* *}\right)$ that $c$ satisfies the requirement. 
THEOREM 5. Let $K_{1}$ and $K_{2}$ be compact convex sets, and $F_{1}$ and $F_{2}$ closed faces of $K_{1}$ and $K_{2}$ respectively. Let $F$ be the face $\overline{\mathrm{co}}\left(\omega\left(F_{1} \times F_{2}\right)\right)$ in $K_{1} \otimes K_{2}$. Then the following holds

(i) If $F$ is a split face of $K_{1} \otimes K_{2}$ then $F_{1}$ and $F_{2}$ are split faces of $K_{1}$ and $K_{2}$.

(ii) If either $A\left(F_{1}\right)$ or $A\left(F_{2}\right)$ has the the approximation property, and $F_{1}$ and $F_{2}$ are split faces of $K_{1}$ and $K_{2}$, then $F$ is a split face of $K_{1} \otimes K_{2}$.

Proof. To prove (i) we assume that $F$ is a split face. As noted before $\partial_{e} F=\omega\left(\partial_{e} F_{1} \times \partial_{e} F_{2}\right)$. Let $a \in A\left(K_{1}\right)$ such that $a \geqq 0$ on $F_{1}$, i.e. $\left.a\right|_{F_{1}} \in A\left(F_{1} ; K_{1}\right)^{+}$. By Proposition 3 it will suffice to show that $\left(a \cdot \chi_{F_{1}}\right)^{\wedge}$ is affine $K_{1}$. We know that $\left((a \otimes 1) \cdot \chi_{F}\right)^{\wedge}$ is u.s.c. and affine on $K_{1} \otimes$ $K_{2}$, since $a \otimes 1$ is nonnegative on $\omega\left(F_{1} \times F_{2}\right)$ and hence on $F$. Now we fix $x_{2} \in \partial_{e} F_{2}$. Then the function $g\left(x_{2}\right): x \rightarrow\left((\alpha \otimes 1) \cdot \chi_{F}\right)^{\wedge}\left(\omega\left(x, x_{2}\right)\right)$ is u.s.c. and affine on $K_{1}$. On $F_{1} g\left(x_{2}\right)$ agrees with $a$, and since $\omega\left(\partial_{e} F_{1}^{\prime} \times \partial_{e} F_{2}\right) \subseteq F^{\prime \prime}$, we have that $g\left(x_{2}\right)=0$ on $\partial_{e} F_{1}^{\prime}$

Since $g\left(x_{2}\right)$ and $\left(a \cdot \chi_{F_{1}}\right)^{\wedge}$ agree on $\partial_{e} K_{1}$, and $g\left(x_{2}\right)$ is u.s.c. affine, while $\left(a \cdot \chi_{F_{1}}\right)^{\wedge}$ is u.s.c. concave it follows from Bauers principle [5; Lem. 1] that $g\left(x_{2}\right) \leqq\left(a \cdot \chi_{F_{1}}\right)^{\wedge}$. Moreover $g\left(x_{2}\right) \geqq a \cdot \chi_{F_{1}}$, and since $\left(a \cdot \chi_{F_{1}}\right)^{\wedge}$ is the smallest u.s.c. concave majorant of $a \cdot \chi_{F_{1}}$, we have $g\left(x_{2}\right) \geqq$ $\left(a \cdot \chi_{F_{1}}\right)^{\wedge}$, and (i) follows.

To prove (ii) we shall assume that $F_{1}$ and $F_{2}$ are split faces, and that $A\left(F_{1}\right)$ has the approximation property. By Proposition 3 we have to show that if $a \in A(F)^{+}$then $a$ admits an extension $\widetilde{a} \in A_{s}\left(K_{1} \otimes K_{2}\right)$ such that $\tilde{a}=0$ on $F^{\prime \prime}$. Now $a \circ\left(\left.\omega_{K_{1} \times K_{2}}\right|_{F_{1} \times F_{2}}\right)$ belongs to $B A\left(F_{1} \times F_{2}\right)=$ $A\left(F_{1}\right) \otimes_{\varepsilon} A\left(F_{2}\right)$. If $\varepsilon>0$ is arbitrary we can choose $a_{1}, \cdots, a_{n} \in A\left(F_{1}\right)$ and $b_{1}, \cdots, b_{n} \in A\left(F_{2}\right)$ such that

$$
\left\|a \circ \omega_{K_{1} \times K_{2}}-\sum_{i=1}^{n} a_{i} \otimes b_{i}\right\|_{F_{1} \times F_{2}}<\varepsilon .
$$

By Proposition 3 we can choose $\widetilde{a}_{i} \in A_{s}\left(K_{1}\right), \widetilde{b}_{i} \in A_{s}\left(K_{2}\right)$ such that $\widetilde{a}_{i}=a_{i}$ on $F_{1}$ and $\tilde{a}_{i}=0$ on $F_{1}^{\prime}$, while $\widetilde{b}_{i}=b_{i}$ on $F_{2}$ and $\widetilde{b}_{i}=0$ on $F_{2}^{\prime}$.

By Lemma $4 \sum_{i=1}^{n} \tilde{a}_{i} \otimes \tilde{b}_{i} \in A_{s}\left(K_{1} \otimes K_{2}\right)$ and on $\omega\left(F_{1} \times F_{2}\right)$ it equals $\sum_{i=1}^{n} a_{i} \otimes b_{i}$, while $\sum_{i=1}^{n} \widetilde{a}_{i} \otimes \widetilde{b}_{i}=0$ on $\partial_{e}\left(K_{1} \otimes K_{2}\right) \backslash \partial_{e} F$.

As $A_{s}\left(K_{1} \otimes K_{2}\right)$ is complete in $\|\quad\|_{\partial_{e}\left(K_{1} \otimes K_{2}\right)}$ and the norm of $\sum_{i=1}^{n} \tilde{a}_{i} \otimes \tilde{b}_{i}$ is obtained at $\omega\left(F_{1} \times F_{2}\right)$, this argument leads to the existence of $\tilde{a} \in A_{s}\left(K_{1} \otimes K_{2}\right)$ such that $\tilde{a}=a$ on $\omega\left(F \times F_{2}\right)$, and $\tilde{a}=0$ on $\partial_{e} F^{\prime}=\partial_{e}\left(K_{1} \otimes K_{2}\right) \backslash F$. It remains to show that $\widetilde{a}=a$ on $F$ and $\widetilde{a}=0$ on $F^{\prime}$.

Now let $x \in F$ and represent $x$ by a probability measure $\mu$ on $\omega\left(F_{1} \times F_{2}\right)$. Since $\widetilde{a}$ satisfies the barycentric calculus we get

$$
\tilde{a}(x)=\int_{K_{1} \otimes K_{2}} \tilde{d} d \mu=\int_{w\left(F_{1} \times F_{2}\right)} \tilde{a} d \mu=\int_{F} a d \mu=a(x)
$$


and so $\widetilde{a}=a$ on $F$.

To show that $\tilde{a}=0$ on $F^{\prime}$ we let $b \in A\left(K_{1} \otimes K_{2}\right)$ with $b>0$ on $K_{1} \otimes K_{2}$ and $b>a$ on $F$. Then $b \geqq \widetilde{a}$ on $\partial_{e}\left(K_{1} \otimes K_{2}\right)$, and by Lemma $2(i), b \geqq \widetilde{a}$ on $K_{1} \otimes K_{2}$. For $\rho \in K_{1} \otimes K_{2}$ we have

$$
\left(a \cdot \chi_{F}\right)^{\wedge}(\rho)=\inf \left\{b(\rho) \mid b \in A\left(K_{1} \otimes K_{2}\right), b>a \cdot \chi_{F}\right\} \geqq \widetilde{\alpha}(\rho) \geqq 0 .
$$

Since $\left(a \cdot \chi_{F}\right)^{\wedge}=0$ on $F^{\prime}$, we get $\widetilde{a}=0$ on $F^{\prime \prime}$, and the proof is complete.

REMARK. It is easy to see from Lemma 4 that the embedding of the product of two parallel faces $F_{1}$ and $F_{2}$ in the sense of [11] gives rise to a parallel face $F$ without the assumption of the presence of the approximation property in $A\left(F_{1}\right)$. In fact, $\hat{\chi}_{F}=\hat{\chi}_{F_{1}} \otimes \hat{\chi}_{F_{2}}$ is affine.

THEOREM 6. Let $F$ be a closed split face of a compact convex set $K$. Let $B$ be a real Banach space having the approximation property. Let $p$ be a concave l.s.c. strictly positive real function on $K$. Let $a: F \rightarrow B$ be an affine continuous map such that

$$
\|a(k)\| \leqq p(k), \text { all } k \in F \text {. }
$$

Then a has an extension to a continuous affine map $\widetilde{a}: K \rightarrow B$ such that

$$
\|\widetilde{a}(k)\| \leqq p(k), \text { all } k \in K
$$

Proof. Let $C$ be the unit ball of $B^{*}$ with $w^{*}$-topology. $B \times \boldsymbol{R}$ is normed by $\|(x, r)\|=\|x\|+|r|$. It was observed in [10] that $(x, r) \rightarrow$ $(\cdot)(x)+r$ is an isometric isomorphism of $B \times \boldsymbol{R}$ onto $A(C)$. Hence if $B$ has the approximation property then $A(C)$ has.

We define a biaffine continuous function $b$ on $F \times C$ by

$$
b\left(x, x^{*}\right)=x^{*}(a(x)), \text { all } x \in F, x^{*} \in C .
$$

By Proposition 1 (ii) there is an affine homeomorphism between $F \otimes C$ and $\overline{\operatorname{co}}\left(\omega_{K \times C}(F \times C)\right)$ defined by

$$
T(\rho)(d)=\rho\left(\left.d\right|_{F \times C}\right) \text { for } d \in B A(K \times C) .
$$

Since $b$ is naturally a continuous affine function on $F \otimes C$ there is a continuous affine function $b_{1}$ on $\overline{c o}\left(\omega_{K \times C}(F \times C)\right)$ such that

$$
b_{1}\left(T \omega_{F \times C}\left(x, x^{*}\right)\right)=x^{*}(a(x)) \text {, all }\left(x, x^{*}\right) \in F \times C .
$$

Moreover $\rho \rightarrow p\left(P_{1}(\rho)\right)$ is concave, strictly positive and 1.s.c. on $K \otimes C$. For $\rho \in \partial_{e}\left(\operatorname{co}\left(\omega_{K \times o}(F \times C)\right)\right)=\omega_{K \times C}\left(\partial_{e} F \times \partial_{e} C\right)$ we have $\rho=$ $\omega_{K \times C}\left(x, x^{*}\right)$ with $\left(x, x^{*}\right) \in \partial_{e} F \times \partial_{e} C$ and hence

$$
\left|b_{1}(\rho)\right|=\left|x^{*}(a(x))\right| \leqq\|a(x)\| \leqq p(x)=p\left(P_{1}(\rho)\right) .
$$


Since $\rho \rightarrow\left|b_{1}(\rho)\right|$ is convex and continuous and $\rho \rightarrow p\left(P_{1}(\rho)\right)$ is concave and 1.s.c., it follows from Bauers principle [5; Lem. 1] that $\left|b_{1}\right| \leqq p \circ P_{1}$ on $\overline{\operatorname{co}}\left(\omega_{K \times C}(F \times C)\right)$.

Now it follows from Theorem 5 that $\overline{\mathrm{co}}\left(\omega_{K \times C}(F \times C)\right)$ is a split face of $K \otimes C$. By [1; Th. II. 6. 12] and [3; Th. 2.2 and Th. 4.5] it follows that there is a function $c \in A(K \otimes C)$ such that $c$ extends $b_{1}$ and

$$
|c(\rho)| \leqq p\left(P_{1}(\rho)\right) \text {, all } \rho \in K \otimes C .
$$

(Actually, it follows from [1; Cor. I. 5.2] that a concave l.s.c. function on a compact convex set is $A(K)$-superharmonic in the sense of [3]. Moreover it should be remarked that the theorems 2.2 and 4.5 of [3] are stated for complex spaces, but the proofs hold almost unchanged for the real case.)

Now we can define a continuous affine map $c_{1}: K \rightarrow A(C)$ by

$$
c_{1}(k)(\cdot)=c(\omega(k, \cdot)) \text {. }
$$

Then for $k \in K$

$$
\left.\left\|c_{1}(k)\right\|=\sup _{x^{*} \in C}\left\|c\left(\omega\left(k, x^{*}\right)\right)\right\| \leqq \sup p\left(P_{1}\left(k, x^{*}\right)\right)\right)=p(k) .
$$

By composing the isometry $S$ between $A(C)$ and $B \times \boldsymbol{R}$ with the canonical projection $Q$ from $B \times \boldsymbol{R}$ to $B$, which has norm 1. we get an affine continuous map $\widetilde{a}\left(=Q \circ S \circ c_{1}\right)$ of $K$ into $B$ such that

$$
\|\widetilde{a}(k)\|=\left\|\left(Q \circ S \circ c_{1}\right)(k)\right\| \leqq\left\|c_{1}(k)\right\| \leqq p(k)
$$

for all $k \in K$. Moreover, for $k \in F, x^{*} \in C$

$$
\begin{aligned}
& x^{*}(\widetilde{a}(k))=x^{*}\left(\left(Q \circ S \circ c_{1}\right)(k)\right)=c_{1}(k)\left(x^{*}\right) \\
= & c\left(\omega\left(k, x^{*}\right)\right)=b_{1}\left(\omega\left(k, x^{*}\right)\right)=x^{*}(a(k)) .
\end{aligned}
$$

Hence for $k \in F: \widetilde{a}(k)=a(k)$.

CoRollary. Let $F$ be a closed split face of a compact convex set $K$. Let $B$ be a real Banach space having the approximation property. Let $a: F \rightarrow B$ be a continuous affine map. Then a admits an extension to a continuous affine function $\widetilde{a}: K \rightarrow B$ such that $\max _{k \in F}\|a(k)\|=$ $\max _{k \in K}\|\widetilde{a}(k)\|$.

REMARK. Conclusions similar to those of Theorem 6 and the Corollary hold with no assumptions on $B$, if instead we know that $A(F)$ has the approximation property. This is f.ex. the case, if $K$ is a simplex. 


\section{REFERENCES}

1. E. M. Alfsen, Compact convex sets and boundary integrals, Ergebnisse der Mathematik, Springer Verlag, Germany, 1971.

2. E. M. Alfsen and T. B. Andersen, Split faces of compact convex sets, Proc. London Math. Soc., 21 (1970), 415-442.

3. E. M. Alfsen and B. Hirsberg, On dominated extensions in linear subspaces of $C_{C}(X)$, Pacific J. of Math. 36 (1971), 567-584.

4. T. B. Andersen, On dominated extension of continuous affine functions on split faces, (to appear in Math. Scand.)

5. H. Bauer, Kennzeichnung kompakter Simplexe mit abgeschlossener Extremalpunkt menge, Archiv der Mathematik, 14 (1963), 415-421.

6. E. Behrends and G. Wittstock, Tensorprodukte kompakter konvexer Mengen, Inventiones Math., 10 (1970), 251-266.

7. F. Combes, Quelques propriétés des $C^{*}$-algèbres, Bull. Sci. Math., 94 (1970), 165-192.

8. U. Krause, Der Satz von Choquet als ein abstrakter Spektralsatz und vice versa, Math. Ann., 184 (1970), 275-296.

9. A Lazar, Affine products of simplexes, Math. Scand., 22 (1968), 165-175.

10. I. Namioka and R. R. Phelps, Tensor products of compact convex sets, Pacific J. Math., 31 (1969), 469-480.

11. M. Rogalski, Topologies faciales dans les convexes compacts, calcul fonctionnel et decomposition spectrale dans le centre d'un espace $A(X)$, Seminaire Choquet, 1969-70, No. 4.

12. Z. Semadeni, Categorical methods in convexity, Proc. Colloq. on Convexity, Copenhagen 1965, 281-307.

Received March 30, 1971. This work was in part supported by the Danish Science Council, J. No. 511-630. The author wants to thank E. Alfsen for helpful comments.

UNIVERSITY OF OSLO

AND

UNIVERSITY OF ÅRHUS 



\section{PACIFIC JOURNAL OF MATHEMATICS}

\section{EDITORS}

H. SAMELSON

Stanford University

Stanford, California 94305

C. R. HobBY

University of Washington

Seattle, Washington 98105
J. DUGUNDJI

Department of Mathematics

University of Southern California

Los Angeles, California 90007

RICHARD ARENS

University of California

Los Angeles, California 90024

\section{ASSOCIATE EDITORS}

E. F. BECKENBACH

B. H. NeumanN

F WoLF

K. YoshidA

\section{SUPPORTING INSTITUTIONS}

UNIVERSITY OF BRITISH COLUMBIA

UNIVERSITY OF SOUTHERN CALIFORNIA

CALIFORNIA INSTITUTE OF TECHNOLOGY

UNIVERSITY OF CALIFORNIA

MONTANA STATE UNIVERSITY

STANFORD UNIVERSITY

UNIVERSITY OF NEVADA

NEW MEXICO STATE UNIVERSITY

OREGON STATE UNIVERSITY

UNIVERSITY OF OREGON

OSAKA UNIVERSITY

UNIVERSITY OF TOKYO

UNIVERSITY OF UTAH

WASHINGTON STATE UNIVERSITY

UNIVERSITY OF WASHINGTON

$\stackrel{*}{*} \stackrel{*}{*} \stackrel{*}{*}{ }^{*}{ }^{*}$ MMEICAN MATHEMATICAL SOCIETY

NAVAL WEAPONS CENTER

Printed in Japan by International Academic Printing Co., Ltd., Tokyo, Japan 


\section{Pacific Journal of Mathematics}

\section{Vol. 42, No. $1 \quad$ January, 1972}

Tage Bai Andersen, On Banach space valued extensions from split faces ........

David Marion Arnold, A duality for quotient divisible abelian groups of finite

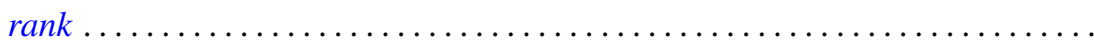

Donald Pollard Ballou, Shock sets for first order nonlinear hyperbolic

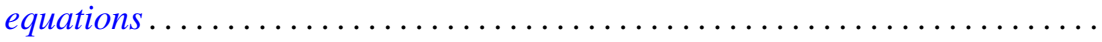

Leon Brown and Lowell J. Hansen, On the range sets of $H^{p}$ functions .........

Alexander Munro Davie and Arne Stray, Interpolation sets for analytic

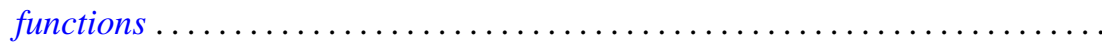

M. G. Deshpande, Structure of right subdirectly irreducible rings. II . . . . . . . . .

Barry J. Gardner, Some closure properties for torsion classes of abelian

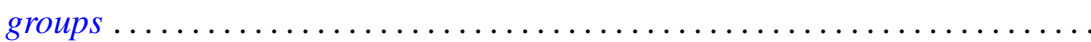

Paul Daniel Hill, Primary groups whose subgroups of smaller cardinality are

direct sums of cyclic groups . . . . . . . . . . . . . . . . . . .

Richard Allan Holzsager, When certain natural maps are equivalences .........

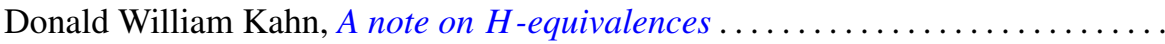

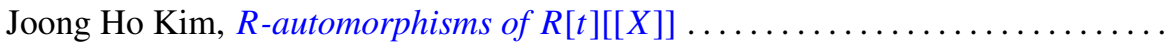

Shin'ichi Kinoshita, On elementary ideals of polyhedra in the 3-sphere.........

Andrew T. Kitchen, Watts cohomology and separability...

Vadim Komkov, A technique for the detection of oscillation of second order

ordinary differential equations .

Charles Philip Lanski and Susan Montgomery, Lie structure of prime rings of characteristic 2

Andrew Lenard, Some remarks on large Toeplitz determinants . .

Kathleen B. Levitz, A characterization of general Z.P.I.-rings. II .

Donald A. Lutz, On the reduction of rank of linear differential systems

David G. Mead, Determinantal ideals, identities, and the Wronskian ...

Arunava Mukherjea, A remark on Tonelli's theorem on integration in product

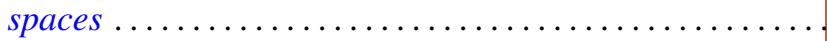

Hyo Chul Myung, A generalization of the prime radical in nonassociative rings.

John Piepenbrink, Rellich densities and an application to unconditionally nonoscillatory elliptic equations.

Michael J. Powers, Lefschetz fixed point theorems for a new class of multi-valued maps .

Aribindi Satyanarayan Rao, On the absolute matrix summability of a Fourier

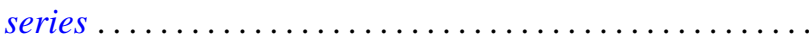

T. S. Ravisankar, On Malcev algebras ......................... 227

William Henry Ruckle, Topologies on sequences spaces . . . . . . . . . . . . . 235

Robert C. Shock, Polynomial rings over finite dimensional rings . . . . . . . . . 251

Richard Tangeman, Strong heredity in radical classes . . . . . . . . . . . . . . 259

B. R. Wenner, Finite-dimensional properties of infinite-dimensional spaces . . . . 267 